\title{
28 Research Square \\ Implementation of Real Time Hybrid Simulation Based On GPU Computing
}

\section{Zhenyun Tang}

Beijing University of Technology

Xiaohui Dong ( $\nabla$ dongxiaohui@emails.bjut.edu.cn )

Beijing University of Technology https://orcid.org/0000-0001-8268-6453

\section{Zhenbao Li}

Beijing University of Technology

\section{Xiuli Du}

Beijing University of Technology

\section{Research Article}

Keywords: graphics processing unit, real time hybrid simulation, numerical integration algorithm, shaking table, finite element analysis

Posted Date: October 1st, 2021

DOl: https://doi.org/10.21203/rs.3.rs-596198/v2

License: (c) (1) This work is licensed under a Creative Commons Attribution 4.0 International License. Read Full License 


\section{Abstract}

With combination of physical experiment and numerical simulation, real-time hybrid simulation (RTHS) can enlarge the dimensions of testing specimens and improve the testing accuracy. However, due to the limitation of computing capacity, the maximum degrees of freedom for numerical substructure are less than 7000 from the reported RTHS testing. It cannot meet the testing requirements for evaluating the dynamic performance of large and complex engineering structures. Taking advantages of parallel computing toolbox (PCT) in Matlab and high-performance computing of graphics processing unit (GPU). A RTHS framework based on MATLAB and GPU was established in this work. Using this framework, a soil-structure interaction system (SSI) was tested by a shaking table based RTHS. Meanwhile, the dynamic response of this SSI system was simulated by finite element analysis. The comparison of simulation and testing results demonstrated that the proposed testing framework can implement RTHS testing successfully. Using this method, the maximum degrees of freedom for numerical substructure can reach to 27,000 , which significantly enhance the testing capacity of RTHS testing for large and complex engineering structures.

\section{Introduction}

Real-time hybrid simulation(RTHS) is a testing method combining physical experiments and numerical calculations[1, 2]. It divides the integral structure into physical and numerical substructure. The former one is tested by physical experiment, and the latter one is simulated by numerical analysis. The coordination of interface responses between two substructures are transferred in real-time. RTHS testing makes it possible to evaluate the dynamic performance of large and complex engineering structures on existing facilities [3].

Generally, the dynamic solution needs to be completed in a very small integration time step (at least 20 $\mathrm{ms}$ ). Efficient explicit integration algorithms, such as the central difference method, are usually used to solve the response of the numerical substructure[4]. However, the stability of convergence is a disadvantage of explicit integration algorithm. In order to ensure the stability and accuracy, a smaller integration step (e.g., $\Delta t=1 \mathrm{~ms}$ ) [5] is often required. Correspondingly, the small integration step will limit the calculation scale of numerical substructure, which cannot meet the RTHS needs for evaluating the dynamic performance of large and complex engineering structures[6]. Optimizing the numerical integration algorithm $[7,8]$ and improving the numerical solution efficiency $[6]$ are primary ways to solve the above problems.

To guarantee the stability of numerical solution for RTHS testing, Chang[9], Nakashima[10]studied unconditionally explicit pseudo-dynamic algorithms. These methods provide only explicit target displacement but not explicit target velocity, which cannot consider the velocity-dependent restoring force in RTHS. Wu[7] proposed a target velocity formulation based on the forward difference of the predicted displacements so as to render the above methods explicit for RTHS. Based on existing integration methods, Nakashima[11] proposed to divide the numerical solution into two parts, response analysis task 
(RAT) and signal generation task (SGT), realized RTHS testing with 10 degrees of freedom(DOFs) under $\Delta t=330 \mathrm{~ms}$ or $12 \mathrm{DOFs}$ under $\Delta t=500 \mathrm{~ms}$. Cheng [12] developed a 'hybrid finite element' program by MATLAB, which combined finite element method(FEM) with hybrid structure testing, realized RTHS testing with 122 DOFs under $\Delta t=10 \mathrm{~ms}$. Chae[13] used Hybrid-FEM technology to accomplish a RTHS testing with 514 DOFs under $\Delta t=10 \mathrm{~ms}$. Saouma[14] developed a program named Mercury running on real-time hardware and completed a nonlinear model RTHS testing with 405 DOFs under $\Delta t=10 \mathrm{~ms}$. Zhu[6] used RAT and SGT in two different target computers, realized RTHS testing with 1240 DOFs under $\Delta t=20 \mathrm{~ms}$. Lu[15] used a RTHS calculation system based on Simulink real-time and four-core parallel programming in Windows environment, realized RTHS testing with about 6000 DOFs (Non-Diagonal Damping Matrix Case) under $\Delta t=20 \mathrm{~ms}$. In summary, the research of algorithms and the development of FEM improve the real-time computing capacity of numerical substructure. However, as known from the reported work, the maximum DOFs of the numerical substructure for RTHS is hard to exceed 7000 .

In the research field of solution efficiency, traditional numerical substructure calculations are all based on Central Processing Unit (CPU) in computer. At present, the calculation capacity of CPU conforms to Moore's Law[16]. Due to the limitation of calculation capacity, complex numerical models are difficult to be solved in real-time using small time step. Since 2008, NVIDIA Corporation has used Graphics Processing Unit (GPU) for scientific computing successfully. Compared with CPU, there are more computing units in the GPU, it is more advantageous to use GPU in large-scale numerical calculations[17]. Papadopoulos[18], Gravvanis[19] proved that GPU parallel calculation based on the Compute Unified Device Architecture (CUDA) has higher calculation efficiency than multi-CPU processors in solving large sparse matrix. In the field of engineering, researchers were also using GPU to accelerate calculation and simulation[20, 21]. With combination of GPU parallel and discrete element method (DEM), Mohammad[22] studied displacement slip faulting through granular soils by a method of GPU-based DEM modelling. Durand[23] realized the simulation of contact between rock and concrete 30 times based on GPU (NVIDIA Fermi C2050) faster than CPU (Intel Xeon X5650 2.67 GHz). Lu[24] used GPU to simulate seismic damage of a medium-sized urban area, achieved 39 times faster than $\mathrm{CPU}$, which were similarly priced. GPU in the field of civil engineering calculation has a very high computational efficiency [25-27]. There are several software support GPU acceleration calculation, including finite element software such as ABAQUS, OpenSEES and ANSYS, and mathematical calculation software such as MATLAB[28-31]. Hence, this work attempts to develop a RTHS framework based on MATLAB and GPU, the accuracy and efficiency of GPU (NVIDIA Tesla V100) compared with CPU (Intel Xeon E5-2690 V4) were verified by simulation and RTHS testing.

\section{Gpu-based Rths Testing System}

This work established a testing system framework, using GPU instead of traditional CPU as the computing hardware. There are two problems that need to be solved before establishing a testing system with GPU computing. One is how to realize the real-time signal interaction between two substructures; the other is how to carry out dynamic analysis for large-scale numerical substructure by GPU. 


\subsection{Schemes of framework}

The framework of the testing framework is composed of three parts, as shown in Fig. 1, including numerical substructure solution, signal transmission and physical substructure loading. In RTHS, the efficiency of numerical solution must meet the requirements of real-time loading of the physical substructure, and the time of each dynamic solution step is fixed. The loop structure of time control in LABVIEW[32] and the Parallel Computing Toolbox (PCT) in MATLAB are used in numerical substructure solution. The loop structure of time control ensures the time of numerical substructure solution fixed in each step. The period of the loop structure is adjusted according to the step size of the integration algorithm. The M-file script (a type of MATLAB script file) for solving numerical substructure is imported to the MATLAB script window[33], which is in the loop structure of time control. Parallel Computing Toolbox (PCT) can be used in MATLAB R2014a or updated version[34]. It supports multi-core CPU and GPU parallel computing. PCT can significantly improve the efficiency of large matrix elementary arithmetic and signal processing.

The function of signal transmission part is to ensure data communication between two substructures in RTHS. The digital signal from the numerical solution computer is converted into analog signal, and then transmitted to shaking table. Signal acquisition cards are needed to achieve the conversion of digital signal and analog signal.

In physical substructure part, the interface response of numerical substructure transmits from the signal transmission part into the controller of shaking table. The shaking table applies the interface response to the physical substructure after receiving the command, the sensors measure the response of physical substructure and return to the numerical substructure through the signal transmission part.

\subsection{Solution of numerical substructure}

As mentioned in introduction, there are many ways for dynamic analysis using GPU. Commercial finite element software has pre-processing functions, it can make preparations for dynamic analysis in MATLAB. Parameter matrices of numerical model are extracted from the pre-processing functions of commercial finite element software.

The numerical substructure is modelled by pre-processing of ABAQUS in this work. The steps for extracting parameters of numerical substructure from ABAQUS are as follows.

Step 1, establish the finite element model.

Step 2, output the stiffness and mass parameters: the model parameters saved in the text file with format of 'inp' will be generated in the file directory. Add the following code to the 'inp' file and save it. After resubmitting the job file, total mass and stiffness parameters are obtained, the parameters are not yet in matrix form.

${ }^{*}$ step, name $=$ matrix 
${ }^{*}$ matrix generate,stiffness, mass

*matrix output,stiffness,mass

*end step

Step 3, convert stiffness and mass parameters to matrices: use MATLAB to import and process the parameters file, the mass and stiffness matrices of numerical substructure are obtained.

Only the mass and stiffness parameters can be extracted from ABAQUS. The damping matrix is constructed from the stiffness and mass matrix based on Rayleigh damping as shown in Eq. (1), [C] is the Rayleigh damping matrix, [M]is the mass matrix, and [K] is the stiffness matrix. $a_{0}$ and $a_{1}$ are two proportional parameters, which are determined according to the first two modal frequencies.

$$
[C]=a_{0}[M]+a_{1}[K]
$$

1

Figure 2 shows the flowchart of numerical substructure solving based on GPU and MATLAB. The realtime solution of numerical substructure in GPU-based RTHS testing requires CPU and GPU, in which CPU is for transmission of the interface response and GPU is for solution.

\section{Numerical Verification}

In order to verify the solving capability of GPU, a soil-structure interaction (SSI) system was adopted to be numerical modal, which is solved by GPU and CPU respectively.

\subsection{Parameters of simulation}

As shown in Fig. 3, the upper part of the SSI system is physical substructure, and the soil is numerical substructure. The size of the numerical model is $30 \mathrm{~m} \times 30 \mathrm{~m} \times 15 \mathrm{~m}$, the density is $1 \times 10^{4} \mathrm{~kg} / \mathrm{m}^{3}$, the Young's modulus is $211 \mathrm{MPa}$, and the damping ratio is 0.5 . Viscoelastic boundary is set to simulate far-field soil boundary conditions. The normal stiffness and damping are $20 \mathrm{kN} / \mathrm{m}$ and $1.437 \times 10^{3} \mathrm{kN} /(\mathrm{m} / \mathrm{s})$, The tangential stiffness and damping are $10 \mathrm{kN} / \mathrm{m}$ and $9.45 \times 10^{3} \mathrm{kN} /(\mathrm{m} / \mathrm{s})$. The Kobe seismic wave is adopted as excitation, the peak ground acceleration (PGA) is adjusted to $0.5 \mathrm{~g}$, central difference method and Newmark-beta method are used for numerical dynamic analysis method.

The modal analysis of the numerical substructure was carried out in ABAQUS, the highest order frequency of the numerical substructure is $79.23 \mathrm{rad} / \mathrm{s}$, the stable condition for central difference method is time step $\Delta t \leqslant T_{n} / \Pi=2 / \omega_{n}=25.20 \mathrm{~ms}, T_{n}$ and $\omega_{n}$ are natural periods and frequencies of the structure. In this work, the maximum time step of RTHS is 20 ms, central difference method will be stable. 
In the simulation, the computing capacities of GPU and CPU based on MATLAB were compared, a personal computer (PC) equipped with consumer-grade GPU and a server equipped with professionalgrade GPU. Table 1 shows the hardware configuration parameters of the PC and the server.

Table 1

The configuration parameters of simulation system

\begin{tabular}{|lll|}
\hline System configuration & PC & GPU Server \\
\hline CPU & Intel i5 8300H & Intel Xeon E5-2690 V4 \\
\hline CPU Performance & 4 cores 2.3 GHz & 14 cores 2.6 GHz \\
GPU & NVIDIA GTX 1050 & NVIDIA Tesla V100 \\
GPU Performance & 3.88 GFLOPS & 10.60 TFLOPS \\
RAM & 8 GB & 32 GB \\
Operation System & Windows 10 64-bit & \\
\hline Software Platform & MATLAB 2020ロCUDA 10.2 \\
\hline
\end{tabular}

\subsection{Results of simulation}

In order to compare the calculation efficiency of CPU and GPU, it is necessary to record their time cost in the process. There is a predictive function to record the cost of programs in MATLAB. In the program, 'tic' is added at the beginning of dynamic analysis program, 'toc' is added at the end, the time cost in each step of dynamic analysis is counted. In order to evaluate the calculation capability of GPU in RTHS testing, the different modal DOFs were set by changing mesh sizes. Three numerical substructures with DOFs of 3888, 6591 and 27000 are solved through CPU and GPU respectively, the floating-point precision of the model parameters is single.

Figure 4 shows displacement time histories of the interface solved by central difference method and Newmark-beta method. Displacement histories analysed by central difference method and Newmark-beta method are same. Table 2 shows the time cost of three numerical substructures solved by central difference method and Newmark-beta method on GPU server when time step is $0.001 \mathrm{~ms}$. The time costs of Newmark-beta calculated on GPU are smaller than those on CPU, the calculation of implicit integration algorithm can also be accelerated by GPU, it can also be used in RTHS testing. Calculating the 27000 DOFs model through explicit integration algorithm takes $17 \mathrm{~ms}$, while the implicit integration algorithm takes $37 \mathrm{~ms}$. Explicit integration algorithm is more efficient than implicit integration algorithm and can calculate large scale of numerical substructure models. Central difference method is selected as the dynamic analysis algorithm in this work. 
Table 2

Comparisons of solving times between central difference method and Newmark-beta method (Unit: ms)

\begin{tabular}{|lllll|}
\hline DOFs & \multicolumn{2}{l|}{ Central difference method } & \multicolumn{2}{l|}{ Newmark-beta method } \\
\cline { 2 - 5 } & $T_{C P U}$ & $T_{G P U}$ & $T_{C P U}$ & $T_{G P U}$ \\
\hline 3888 & 17 & 2 & 20 & 3 \\
\hline 6591 & 48 & 4 & 68 & 6 \\
\hline 27000 & 936 & 17 & 1289 & 37 \\
\hline
\end{tabular}

Table 3 shows the central difference method time cost of three numerical substructures and speedup ratio (SR) between CPU and GPU. SR is calculated from Eq. (2). $T_{C P U}$ is the time cost of numerical solution based on CPU in each step, and $T_{G P U}$ is the time cost of that based on GPU.

$$
S R=\frac{T_{C P U}}{T_{G P U}}
$$

When DOFs of numerical model is 3888, the time cost taken by CPU on PC and the CPU on server are similar, it shows the calculation capacity of the CPU on server cannot be brought into full play when the computation is small. When using GPU to solve the model, the speed of the GPU on server is much faster than the GPU on PC. The speed of GPU is 2 times faster than CPU on PC, and the speed of GPU on server is 8.5 times faster than CPU on server. When the DOFs is 6591, the CPU on PC needs $68 \mathrm{~ms}$ in each step, while GPU only needs $21 \mathrm{~ms}$, the speedup effect of GPU on PC is 3.4 times faster than CPU on PC, and the speedup effect of the GPU on server is 12 times faster than CPU on server. When the DOFs is more than 6951, it will exceed the calculation capability of MATLAB on PC, but the DOFs can further increase on the server. 
Table 3

Comparisons of solving times between PC and GPU

Server (Unit: ms)

\begin{tabular}{|lllllll|}
\hline DOFs & PC & \multicolumn{5}{c|}{ GPU Server } \\
\cline { 2 - 7 } & $T_{C P U}$ & $T_{G P U}$ & SR & $T_{C P U}$ & $T_{G P U}$ & SR \\
\hline 3888 & 20 & 10 & 2 & 17 & 2 & 8.5 \\
\hline 6591 & 68 & 21 & 3.4 & 48 & 4 & 12 \\
\hline 27000 & \multicolumn{2}{l}{ Out of GPU memory } & 936 & 17 & 55 \\
\hline
\end{tabular}

With increase of DOFs, the numerical solution for each time step cost more time. In this work, when the time step is $17 \mathrm{~ms}$, the maximum DOFs solved in real time is 27,000 . It needs $936 \mathrm{~ms}$ for the time step of 20 ms to solve this model through the CPU on server. The GPU on server can achieve 55 times faster than the CPU on server. When the DOFs is more than 27,000, it is out of the memory of MATLAB on the server.

Figure 5 shows the displacement time history of the interface between two substructures. when the DOFs of numerical model is 3888, and time steps are $1 \mathrm{~ms}, 5 \mathrm{~ms}$ and $20 \mathrm{~ms}$ respectively. Figure 4 (a) shows the displacement time history solved by CPU and GPU with $\Delta t=1 \mathrm{~ms}$. In Fig. 4(b-d), X-axis is the displacement time history solved by GPU, and Y-axis is the displacement time history solved by CPU. It can be seen from the Fig. 4(b-d) that all of them are straight lines, which show that solution based on GPU has the same accuracy as CPU. GPU can be used instead of CPU to dynamic analysis, and its calculation capability is higher than CPU's when the DOFs is large.

\section{Experimental Verification}

The solving capability of GPU was verified by simulation in Sect. 3, the performance of GPU-based testing system will be evaluated by shaking table RTHS testing in this section.

\subsection{Schemes of testing}

Figure 6 shows the principle of RTHS based on shaking table. The frame structure was installed on the shaking table as physical substructure. It interacted with numerical substructure in real time to test the dynamic performance of integral structure. The schemes of RTHS testing in this work are shown in Fig. 7. GPU was used to solve numerical model, and the solving time of numerical substructure was controlled by the loop structure of time control in LABVIEW. After solving numerical substructure based on GPU, the interface response $y_{N}$ was sent to physical substructure by signal transmission part. The physical substructure was loaded by shaking table after the loading system receiving the response. The dynamic response of the physical substructure $f$ was measured by sensors and transmitted to numerical substructure. The dynamic characteristics of the shaking table were compensated by an out-loop controller, which was added between the signal transmission part and the shaking table. The command 
signal $y_{N}$ was generated by the controller. The out-loop controller program was written in SIMULINK and then downloaded into dSPACE to run in real time.

\subsection{Testing implementation}

The parameters of experiment modal were same as Fig. 3 in Sect. 3.1. In this shaking table RTHS testing, the loading facility is a $0.5 \mathrm{~m} \times 0.5 \mathrm{~m}$ electromagnetic shaking table, solving part of numerical substructure is same as the GPU server configuration in Table 1, and the other parts are shown in Table 4.

Table 4

Testing system configuration parameters

\begin{tabular}{|ll|}
\hline Parts & configuration parameters \\
\hline Signal transmission & NICompactDAQ-9174[NI-9201[NI-9263 \\
\hline Real-time simulation & dSPACE MicroLabBOX-1202 \\
\hline
\end{tabular}

The layout of the physical substructure and the sensors were shown in Fig. 8.During the testing process, the sensors were connected to the control system of the shaking table. The acceleration sensors were installed on the top of the physical substructure and the shaking table. And the displacement of the physical substructure was measured by a laser displacement meter. The displacement of the shaking table was obtained from the control system of the shaking table. The physical substructure is an aluminum single-layer steel frame, and the bottom was fixed on the shaking table by bolts. The top mass of the physical substructure is $7 \mathrm{~kg}$, and the mass of the four struts is $0.48 \mathrm{~kg}$. As the struts mass only accounts for a small part of the total mass, the sum of the $1 / 2$ struts mass and the top mass were added as the mass of the physical substructure, that is $m=7.24 \mathrm{~kg}$. White noise displacement command signal with frequency range of $0.2-15 \mathrm{~Hz}$ and amplitude of $2 \mathrm{~mm}$ was inputted to the shaking table, and the stiffness and damping of the physical substructure are identified as $k=753.25 \mathrm{~N} / \mathrm{m}$ and $c=0.44$ $\mathrm{N} /(\mathrm{m} / \mathrm{s})$. The Kobe and El-Centro waves with $\mathrm{PGA}=0.5 \mathrm{~g}$ were input to shaking table, and the displacement of the physical substructure was compared with integral simulation through central difference method. Figure 9(i) shows the displacement time history results. In Fig. 9(ii), X-axis is the displacement time history in simulation, and $\mathrm{Y}$-axis is the displacement time history of physical substructure in RTHS testing. The inevitable error existed when the frame was treated as a linear timeinvariant system. Therefore, the measured and simulated response in Fig. 9 was not consistent perfectly. However, this work focuses on the feasibility of GPU for RTHS testing. The model of this frame is still accurate enough for evaluating the testing capacity of GPU-based RTHS system.

Furthermore, a white noise displacement signal with frequency range of $0.2-15 \mathrm{~Hz}$ and amplitude of 2 $\mathrm{mm}$ was input to the shaking table, the input signal and displacement of the shaking table were recorded. A fourth-order transfer function was used to identify characteristics of the shaking table, and the transfer function is shown in Eq. (3). 


$$
G_{s t}=\frac{1.023 e 9}{s^{4}+427.5 s^{3}+1.481 e 05 s^{2}+3.041 e 07 s+1.017 e 09}
$$

3

Figure 10 shows the transfer function of the shaking table and identified results. The amplitude and phase errors of the shaking table signals are very small when the frequency between $0-3 \mathrm{~Hz}$, hence the shaking table command can be accurately loaded in this frequency range. When the frequency exceeds 3 $\mathrm{Hz}$, the differences of amplitude and phase increase with the rise of frequency, so it is necessary to add an out-loop controller to improve the accuracy of loading.

Kobe wave with PGA of $0.5 \mathrm{~g}$ as input command of the shaking table. Figure 12 shows the expected time histories and the achieved response with or without FSCS controller. The time histories with FSCS controller are much closer to the expectation than those without FSCS. The results show that the FSCS controlled shaking table is suitable for the RTHS testing.

In this testing framework, LABVIEW and MATLAB used in the numerical substructure were based on Windows operating system (OS). Windows is not a real-time $O S$, the real-time performance is unstable[36], the maximum of Windows clock frequency is $1 \mathrm{kHz}$. In order to ensure that more CPU and memory resources are allocated to the numerical calculation, the priority of LABVIEW and MATLAB can be set higher in Windows OS task manager. There is a delay in data communication when LABVIEW invoked MATLAB, which leaded to a delay of nearly $3 \mathrm{~ms}$ in data transmission between MATLAB and LABVIEW. That is to say, after the dynamic analysis of numerical substructure is completed, it takes $3 \mathrm{~ms}$ to send the data to the signal transmission part. The MATLAB script stops calculating within this $3 \mathrm{~ms}$. Therefore, the minimum time step in the RTHS testing in this work is shown as Eq. (4).

$$
\Delta t=t_{\text {solve }}+3
$$

4

$\Delta t$ in Eq. (4) is the actual time step of numerical integration, and $t_{\text {solve }}$ is the actual time cost. For example, the time step would be $4 \mathrm{~ms}$ when the time cost of solution is $1 \mathrm{~ms}$. The accuracy of numerical integration is greatly affected by the time step[37], hence the maximum time step of RTHS testing is 20 $\mathrm{ms}$ in this work, because of the $3 \mathrm{~ms}$ in data communication, the actual time cost of solution is $17 \mathrm{~ms}$.

\subsection{Testing results}

Table 5 shows the maximum DOFs of the numerical substructure model solved by GPU and CPU, with different time steps using double and single precision. Figure 13 shows time histories of the physical substructure solved by GPU and CPU, with $\Delta \mathrm{t}=4 \mathrm{~ms}$ and double precision data. The results of RTHS testing were consistent with the results of integral simulation, which indicated that the testing framework can meet the accuracy requirements of RTHS testing. Figure 14 shows displacement time histories of physical substructure obtained from GPU-based RTHS testing and integral simulation under working conditions NO.2-9 in Table 5. Groups (i) and (ii) in Figure 14 are $4 \mathrm{~ms}$ and $20 \mathrm{~ms}$ respectively. The displacement time histories of simulation are taken as X-axis, and the displacement time histories of the 
GPU-based RTHS testing are taken as Y-axis. All plots of GPU-based RTHS and simulation are basically linear lines. In some cases, the simulated response cannot match perfectly with RTHS testing results because of the modelling errors of physical substructure shown in Figure 9 but not the calculation errors resulted from GPU or CPU. Therefore, we are still sure that, with the same numerical substructure, the precision of RTHS testing based on GPU is same as CPU.

Table 5 Performance comparisons of numerical solution by GPU and CPU

\begin{tabular}{|lllll|}
\hline NO. & $\Delta \mathrm{t}(\mathrm{ms})$ & Solving hardware & Precision & DOFs \\
1 & 4 & CPU & double & 1500 \\
2 & 4 & GPU & double & 1500 \\
3 & 4 & GPU & single & 3168 \\
4 & 4 & CPU & double & 1080 \\
5 & 4 & GPU & single & 1500 \\
6 & 20 & GPU & double & 18876 \\
7 & 20 & GPU & single & 27000 \\
8 & 20 & CPU & double & 2904 \\
9 & 20 & CPU & single & 3888 \\
10 & 5 & GPU & single & 3888 \\
11 & 20 & GPU & single & 3888 \\
\hline
\end{tabular}

In order to discuss the influence of integration step in GPU-based RTHS testing, a numerical substructure model with 3888 DOFs was solved by GPU and CPU. NO.8 and 9 in Table 5 show that the minimum $\Delta t$ are $5 \mathrm{~ms}$ and $20 \mathrm{~ms}$ respectively. Figure 15 shows displacement and acceleration time histories of physical substructure in the RTHS testing and integral simulation. The peak error of displacement between the RTHS testing and the simulation is $6.74 \%$ when $\Delta t=5 \mathrm{~ms}$, and $13.45 \%$ when $\Delta t=20 \mathrm{~ms}$. The peak error of acceleration between the RTHS testing and the simulation is $6.76 \%$ when $\Delta t=5 \mathrm{~ms}$, and $16.91 \%$ when $\Delta t=20 \mathrm{~ms}$. Therefore, GPU solution can carry out the RTHS testing with smaller time step than CPU solution and improve the accuracy of solution.

\subsection{Discussion of testing results}

NO. $2-5$ in Table 5 shows that, with $\Delta \mathrm{t}=4 \mathrm{~ms}$ and double precision, the maximum DOFs is 1500 solved by GPU and 1080 solved by CPU, the advantage of GPU solving is not obvious. When the precision is single, the maximum DOFs is 3168 solved by GPU, and 1500 solved by CPU. The advance of solution by GPU with single precision is higher than double precision. 
NO. 6-9 in Table 5 shows that, with $\Delta \mathrm{t}=20 \mathrm{~ms}$ and double precision, the maximum DOFs is 18,876 solved by GPU, and 2904 solved by CPU is. When the precision is single, the maximum DOFs is 27,000 solved by GPU, and 3888 solved by CPU. It can be seen that when $\Delta \mathrm{t}=20 \mathrm{~ms}$, the advantage of DOFs solved by GPU is obvious, the maximum DOFs solved by GPU far exceed those solved by CPU. The RTHS testing with large-scale numerical substructure can be realized by GPU solution, which cannot be solved by CPU with the same time step.

In RTHS testing, the size of integration step needs to meet the requirement of real time. Using the GPU server and PCT, the RTHS testing with 27,000 DOFs can be carried out with $\Delta t=20 \mathrm{~ms}$, it needs $926 \mathrm{~ms}$ to solve by CPU at least. Solution by CPU is far from meeting the real time requirement of numerical solution. When the numerical substructure is 3888 DOFs, the time step is reduced from $20 \mathrm{~ms}$ (CPUbased) to $5 \mathrm{~ms}$ (GPU-based). According to results of the different time steps, the solution accuracy can be improved with smaller time step. The time step can be reduced, and the testing accuracy can be improved through the GPU-based solution in RTHS testing.

Due to the instability of elock frequeney in Windows 0 s and the time delay eaused by eommunieation, Duo to the minimum execution time of task is $1 \mathrm{~ms}$ on Windows OS and the time delay caused by communication between LABVIEW and MATLAB, when $\Delta \mathrm{t}$ is $4 \mathrm{~ms}$, the actual time cost of solution is 1 ms. The performance of GPU-based RTHS testing is limited. The maximum DOFs is 27,000 in the GPUbased RTHS testing because of the limitation of MATLAB in this work. Hence, the method of GPU solution, GPU parallel calculation, resource allocation and data communication still can be optimized, which may further improve the scale and efficiency of the solutions.

\section{Conclusion}

The advantage of RTHS is to combine physical testing with numerical simulation and improve the testing capability of existing equipment. Due to the limitations of calculation cost, only large integration time step (e.g., $20 \mathrm{~ms}$ ) and less DOFs (e.g., 7000) for numerical substructure solutions are allowed in the current RTHS testing. It is detrimental to utilize the testing capability and accuracy of RTHS testing. In order to overcome these drawbacks, this work established a RTHS framework based on GPU and Matlab. The performance of framework was verified by numerical simulation and experimental testing. The following conclusions were drawn.

1. Under the condition of $\Delta t=20 \mathrm{~ms}$ and single precision data, using the RTHS testing framework based on MATLAB and GPU (NVIDIA Tesla V100, 10.60 TFLOPS), the maximum DOFs for numerical substructure can reach 27,000 in real-time solution, the speed of GPU calculation is 55 times faster than that of CPU (Intel Xeon E5-2690 V4, 14 cores $2.6 \mathrm{GHz}$ ). And using this framework the numerical substructure based on CPU can only reach 3888 with $\Delta t=20 \mathrm{~ms}$ in real-time solution. The testing capacity of RTHS is significantly enhanced by GPU solving.

2. With the numerical substructure of 3888 DOFs and the model parameters are single precision, the minimum $\Delta t$ is $5 \mathrm{~ms}$ by the RTHS testing based on GPU, and that is $20 \mathrm{~ms}$ based on CPU. The RTHS 
testing results are compared with the integral simulation, the peak errors of displacement and acceleration time history are reduced, Using the testing framework based on GPU in this work can reduce the time step and improve the testing accuracy.

3. MATLAB and LABVIEW under Windows operating system are used in this work. Because of instability of Windows clock frequency and delay of communication between two software, it takes 3 ms to transfer the response data, hence the performance of GPU calculation is limited. Further research is needed to consider real-time performance of operating system and optimization of software interaction, it can further decrease the time step and enhance the DOFs of numerical substructures in RTHS testing.

\section{Declarations}

\section{Acknowledgement}

The authors gratefully acknowledge the support of the National Natural Science Foundation of China under grant number 51978016 .

\section{References}

1. Nakashima M, Kato H, Takaoka E (1992) Development of real-time pseudo dynamic testing. Earthquake Engineering Structural Dynamics 21:79-92

2. Mccrum DP, Williams MS (2016) An overview of seismic hybrid testing of engineering structures. Eng Struct 118:240-261

3. Blakeborough A, Williams M, Darby A, Williams D (2001) The development of real-time substructure testing. Philosophical Transactions of the Royal Society of London Series A: Mathematical Physical Engineering Sciences 359:1869-1891

4. Zhu F, Wang JT, Jin F, Gui Y (2016) Comparison of explicit integration algorithms for real-time hybrid simulation. Bull Earthq Eng 14:89-114

5. Wang M, Au FTK (2006) Assessment and improvement of precise time step integration method. Comput Struct 84:779-786

6. Zhu F, Wang J, Jin F, Zhou M, Gui Y (2014) Simulation of large-scale numerical substructure in realtime dynamic hybrid testing. Earthquake Engineering Engineering Vibration 13:599-609

7. Wu B, Xu G, Wang Q, Williams MS (2006) Operator-splitting method for real-time substructure testing. Earthquake Engineering Structural Dynamics 35:293-314

8. Wu B, Bao H, Ou J, Tian S (2005) Stability and accuracy analysis of the central difference method for real-time substructure testing. Earthquake Engineering Structural Dynamics 34:705-718

9. Chang S, Sung Y, An enhanced explicit pseudodynamic algorithm with unconditional stability, in: 100th Anniversary Earthquake Conference, 2006 
10. Nakashima M, Integration techniques for substructure pseudo-dynamic test, in: 4th US National Conference on Earthquake Engineering (1990) 5, 1990

11. Nakashima M, Masaoka N (1999) Real-time on-line test for MDOF systems. Earthquake engineering structural dynamics 28:393-420

12. Chen C, Ricles JM (2008) Stability analysis of SDOF real-time hybrid testing systems with explicit integration algorithms and actuator delay. Earthquake Engineering Structural Dynamics 37:597-613

13. Chae Y, Kazemibidokhti K, Ricles JM (2013) Adaptive time series compensator for delay compensation of servo-hydraulic actuator systems for real-time hybrid simulation. Earthquake Engineering Structural Dynamics 42:1697-1715

14. Saouma V, Kang DH, Haussmann G (2012) A computational finite-element program for hybrid simulation. Earthquake engineering structural dynamics 41:375-389

15. Improvement of Real-Time Hybrid Simulation Using Parallel Finite-Element Program (2018) \%J Journal of Earthquake Engineering 24:1-19

16. Schaller RR (1997) Moore's law: past, present and future. IEEE Spectr 34:52-59

17. Kirk D (2007) NVIDIA CUDA software and GPU parallel computing architecture. in: ISMM, pp 103104

18. Filelis-Papadopoulos C, Gravvanis GA, Matskanidis P, Giannoutakis KM (2011) On the GPGPU parallelization issues of finite element approximate inverse preconditioning. J Comput Appl Math 236:294-307

19. Gravvanis GA, Filelis-Papadopoulos C, Giannoutakis KM (2012) Solving finite difference linear systems on GPUs: CUDA based parallel explicit preconditioned biconjugate conjugate gradient type methods. The Journal of Supercomputing 61:590-604

20. Liu Z, Wang Y, Hua X, Zhu HP, Zhu ZW, Optimization of wind turbine TMD under real wind distribution countering wake effects using GPU acceleration and machine learning technologies, Journal of Wind Engineering and Industrial Aerodynamics, 208 (2020)

21. Dazzi S, Vacondio R, Mignosa P (2020) Internal boundary conditions for a GPU-accelerated 2D shallow water model: Implementation and applications. Adv Water Resour 137:103525-

22. Hazeghian M, Soroush $A$ (2017) Numerical modeling of dip-slip faulting through granular soils using DEM. Soil Dynamics Earthquake Engineering 97:155-171

23. Durand M, Marin P, Faure F, Raffin B (2012) DEM-based simulation of concrete structures on GPU. European journal of environmental civil engineering 16:1102-1114

24. Lu X, Han B, Hori M, Xiong C, Xu Z (2014) A coarse-grained parallel approach for seismic damage simulations of urban areas based on refined models and GPU/CPU cooperative computing. Adv Eng Softw 70:90-103

25. Cai Y, Li G, Wang H, Zheng G, Lin S (2012) Development of parallel explicit finite element sheet forming simulation system based on GPU architecture. Adv Eng Softw 45:370-379 
26. Zhang W, Zhong Z-h, Peng C, Yuan W-h, Wu W (2021) GPU-accelerated smoothed particle finite element method for large deformation analysis in geomechanics. Comput Geotech 129:103856

27. Cao X, Cai Y, Cui X (2020) A parallel numerical acoustic simulation on a GPU using an edge-based smoothed finite element method. Adv Eng Softw 148:102835

28. Johnsen SF, Taylor ZA, Clarkson MJ, Hipwell J, Modat M, Eiben B, Han L, Hu Y, Mertzanidou T, Hawkes DJ (2015) NiftySim: A GPU-based nonlinear finite element package for simulation of soft tissue biomechanics. Int J Comput Assist Radiol Surg 10:1077-1095

29. Krawezik GP, Poole G, Accelerating the ANSYS direct sparse solver with GPUs, in: Symposium on Application Accelerators in High Performance Computing, SAAHPC, 2009

30. Lu X, Tian Y, Cen S, Guan H, Xie L, Wang L (2018) A high-performance quadrilateral flat shell element for seismic collapse simulation of tall buildings and its implementation in OpenSees. J Earthquake Eng 22:1662-1682

31. Suh JW, Kim Y, Accelerating MATLAB with GPU computing: A primer with examples, Newnes, 2013

32. Lee SK, Park E, Min KW, Park JH (2007) Real-time substructuring technique for the shaking table test of upper substructures. Eng Struct 29:2219-2232

33. Patrascoiu N (2005) Modeling and simulation of the DC motor using MatLAB and LabVIEW. Int J Eng Educ 21:49-54

34. Ploskas N, Samaras N, GPU programming in MATLAB, Morgan Kaufmann, 2016

35. Tang Z, Dietz M, Hong Y, Li Z, Performance extension of shaking table-based real-time dynamic hybrid testing through full state control via simulation, Structural Control and Health Monitoring, 27 (2020)

36. Lina C, Qiang Z, The design and implementation of real-time HILS based on RTX platform, in: IEEE 10th International Conference on Industrial Informatics, IEEE, 2012, pp. 276-280

37. Wu B, Bao H, Ou J, Tian S (2010) Stability and accuracy analysis of central difference method for real-time substructure testing. Earthquake Engineering Structural Dynamics 34:705-718

\section{Figures}




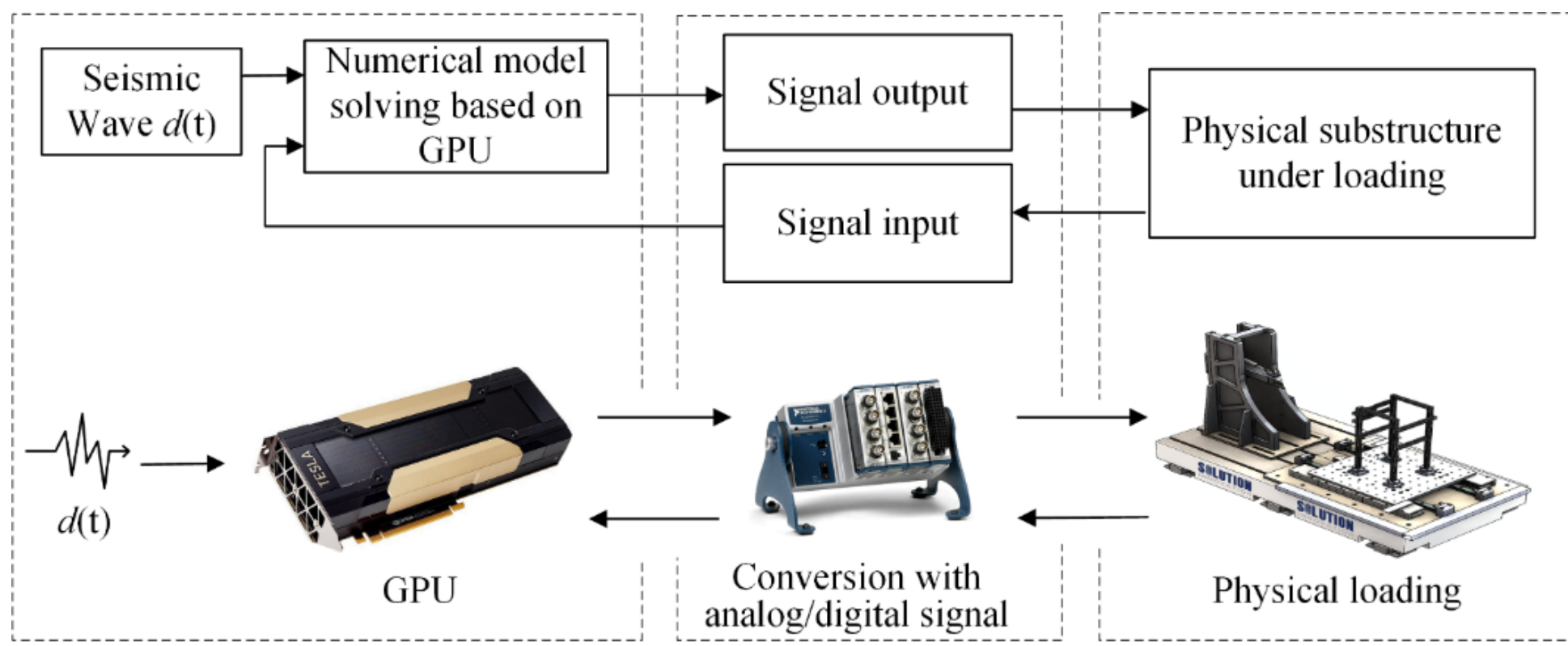

Numerical substructure

Signal transmission

Physical substructure

Figure 1

GPU-based framework for RTHS.

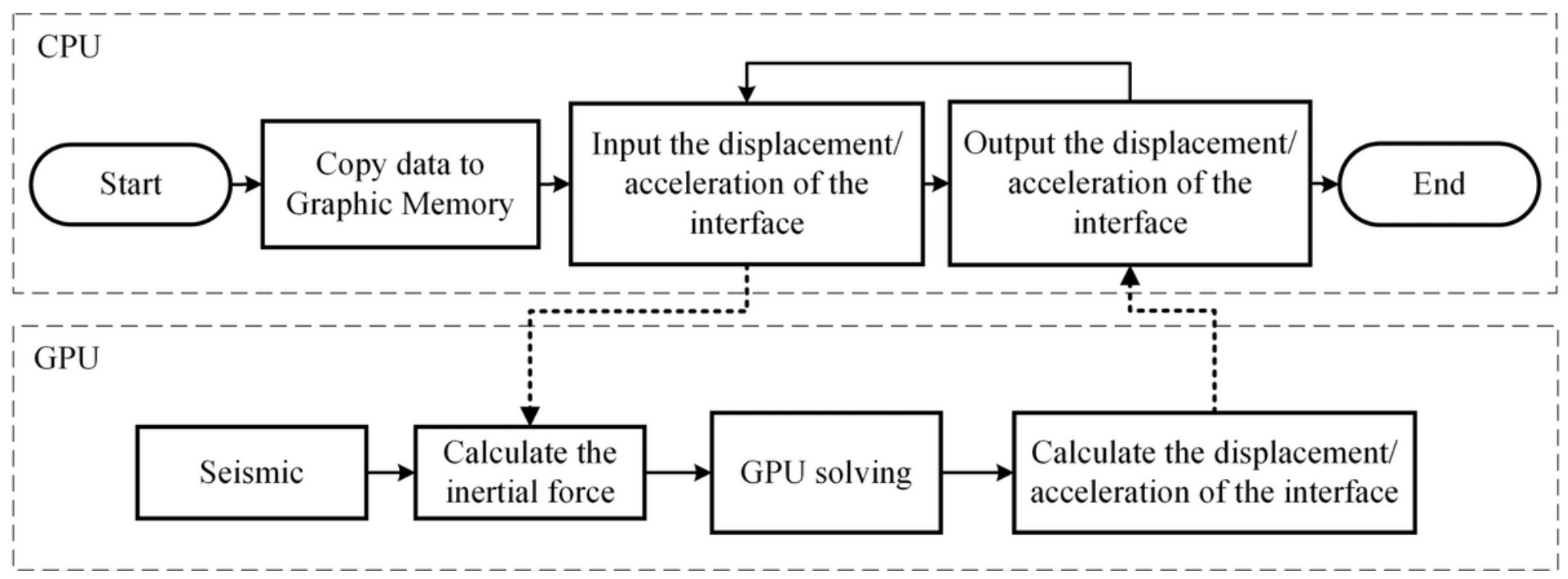

Figure 2

Solution of numerical substructure in GPU-based RTHS testing. 


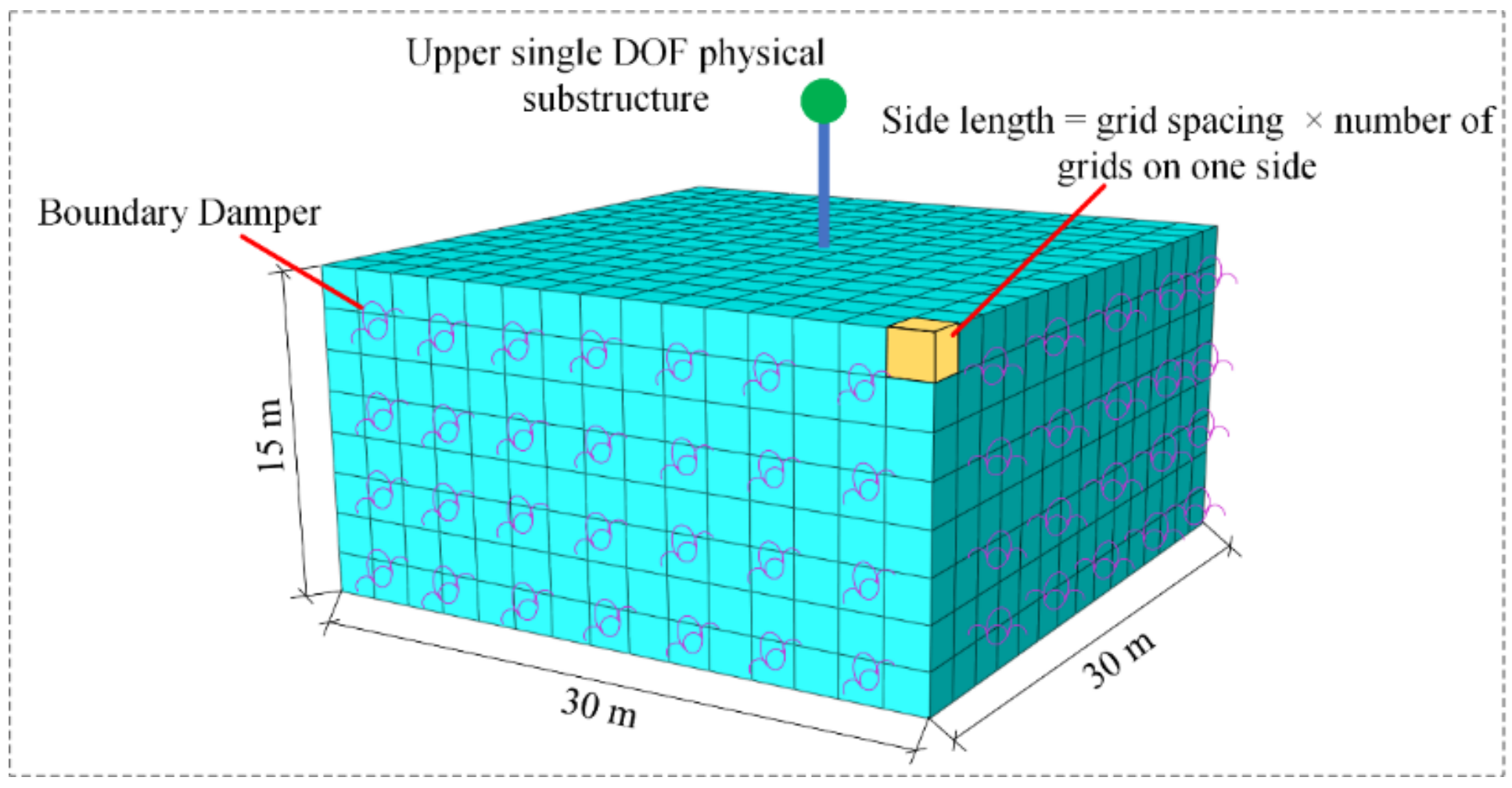

Figure 3

Soil-structure interaction system. 

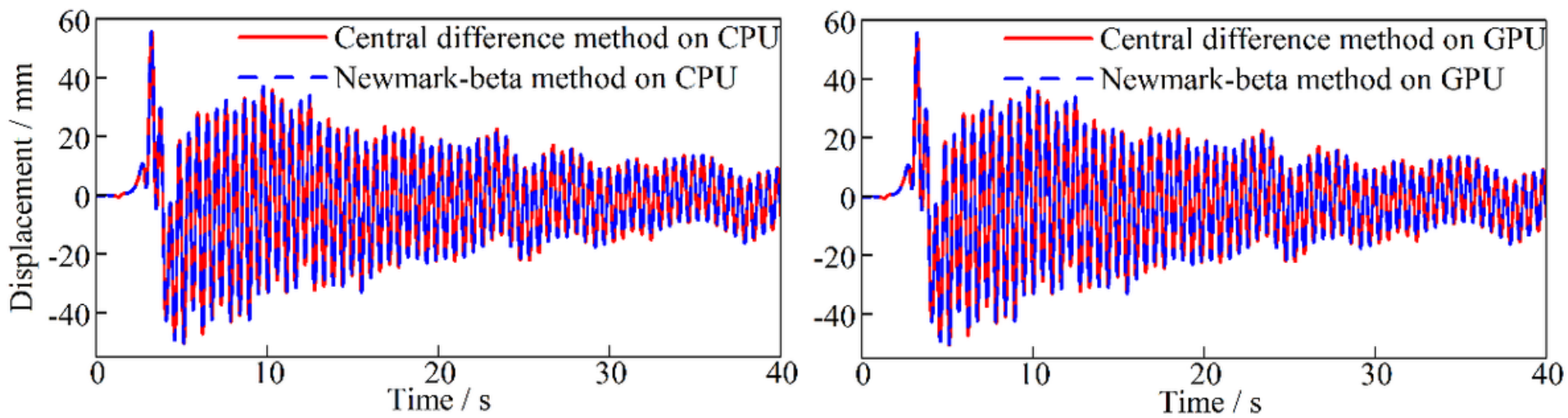

(a) DOFs $=3888$
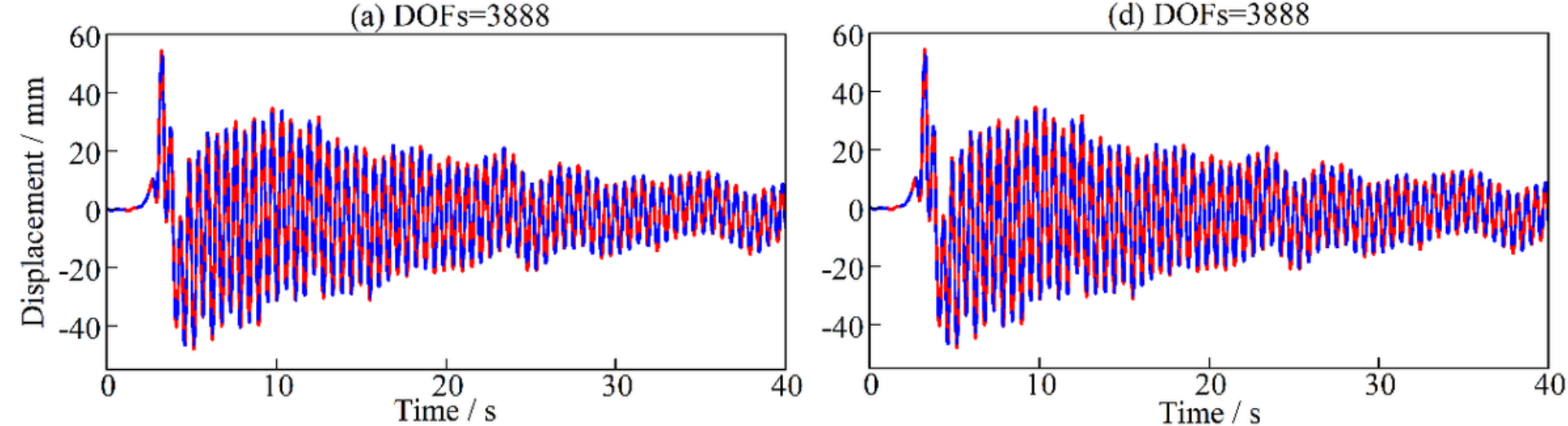

(b) $\mathrm{DOFs}=6591$

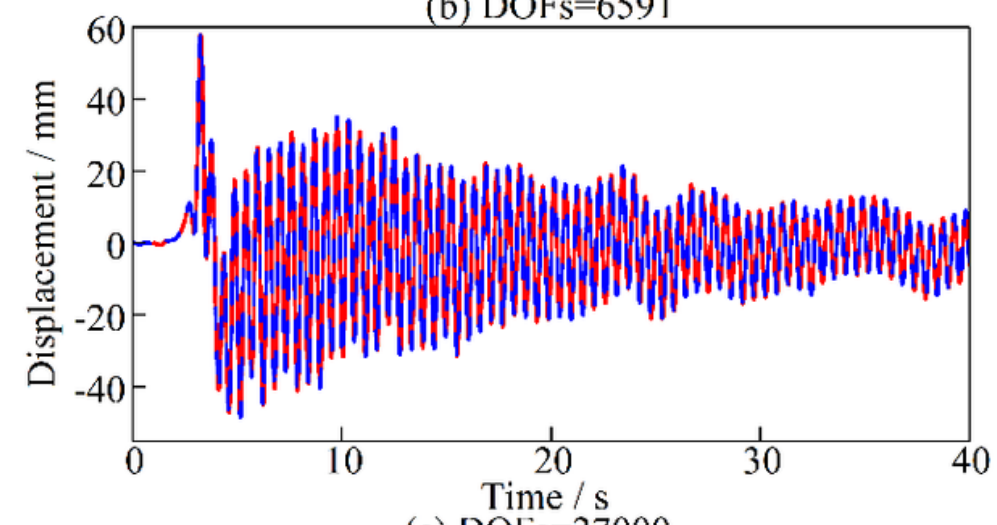

(c) $\mathrm{DOF}_{\mathrm{s}}=6591$

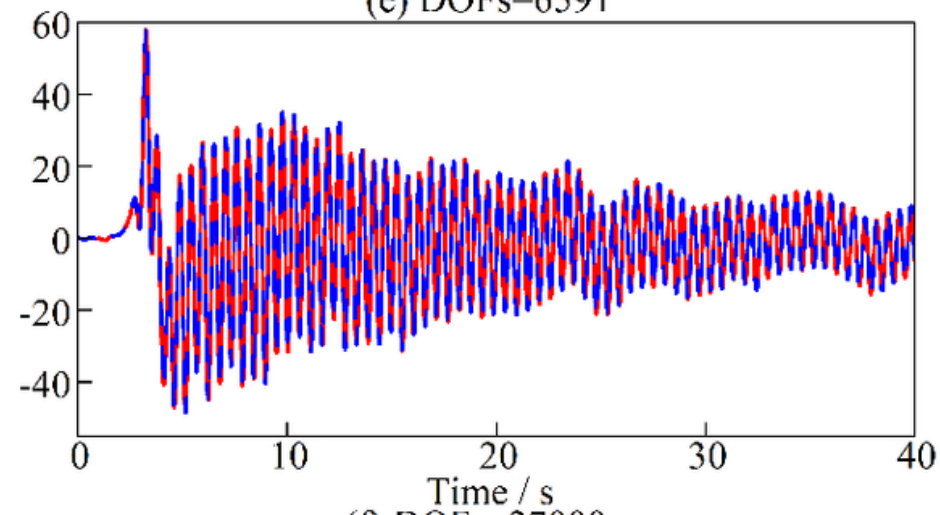

(f) $\mathrm{DOFs}=27000$

Figure 4

Displacement time histories of the interface solved by central difference method and Newmark-beta method 


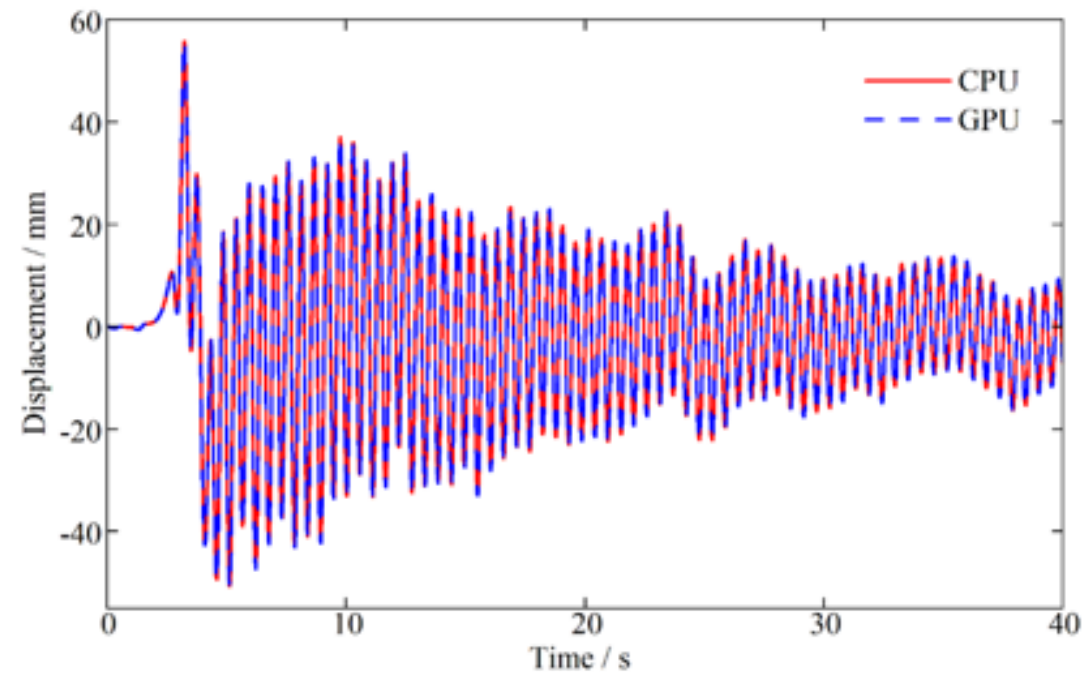

(a) $\Delta t=1 \mathrm{~ms}$

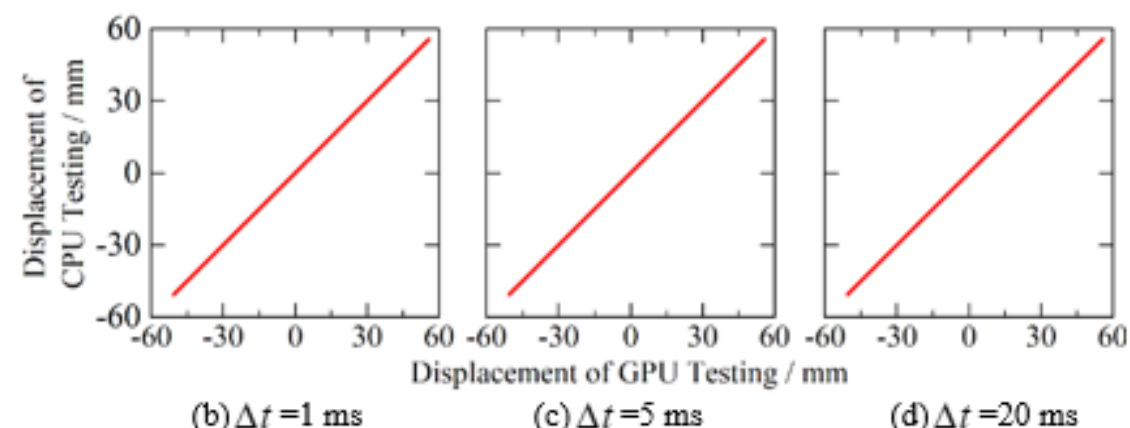

Figure 5

Displacement time histories of the interface based on CPU and GPU 

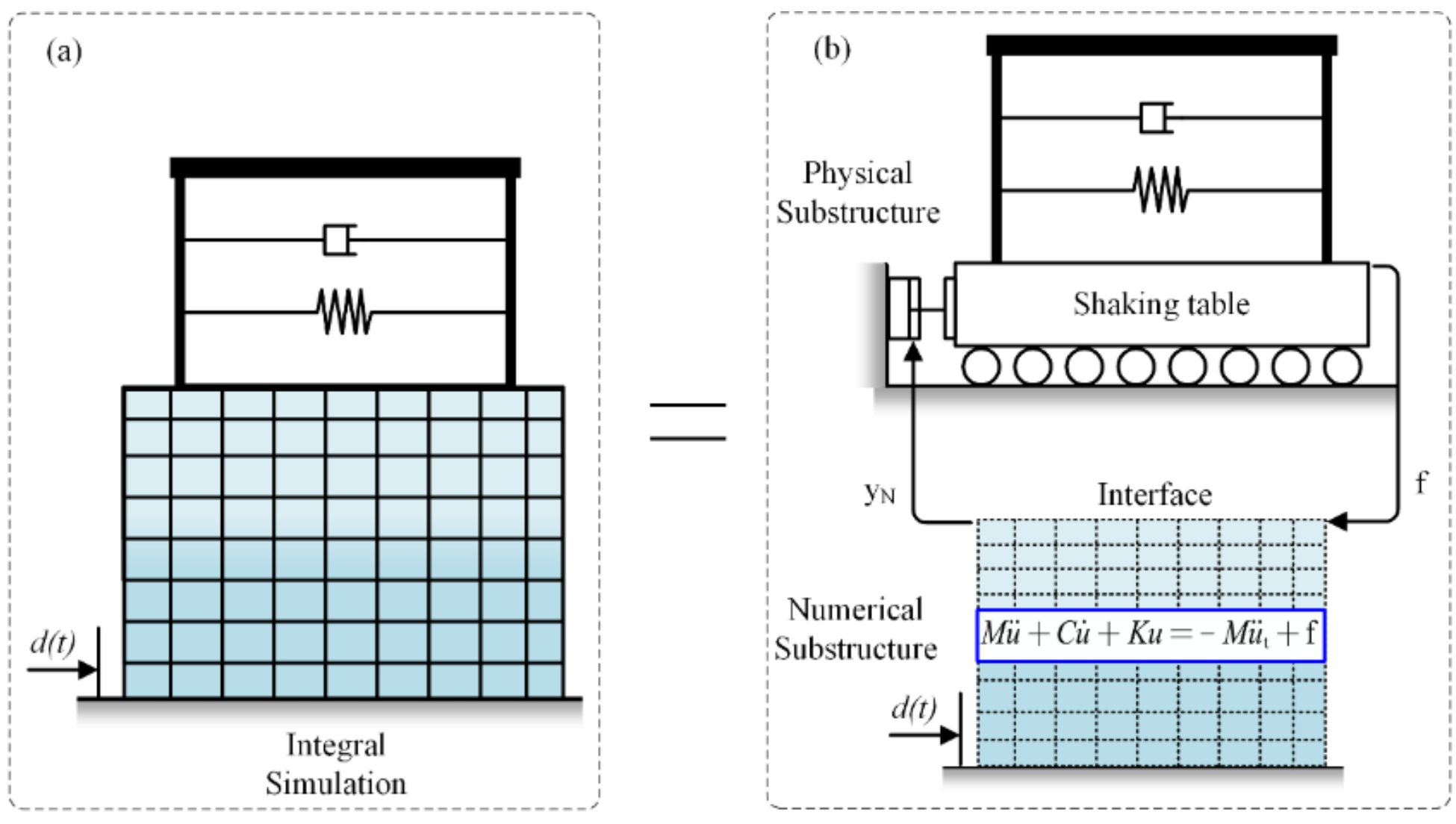

Figure 6

RTHS testing with shaking table

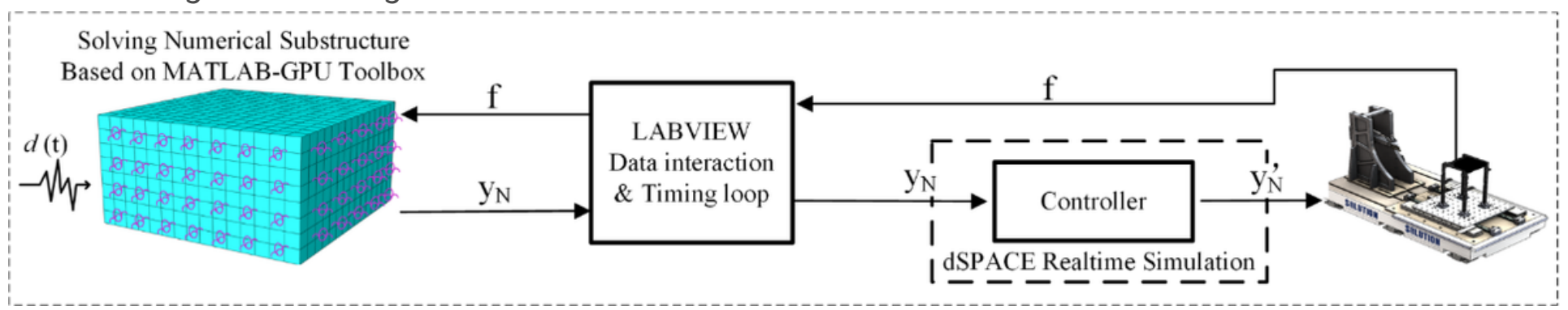

Figure 7

Schemes of the RTHS testing framework 


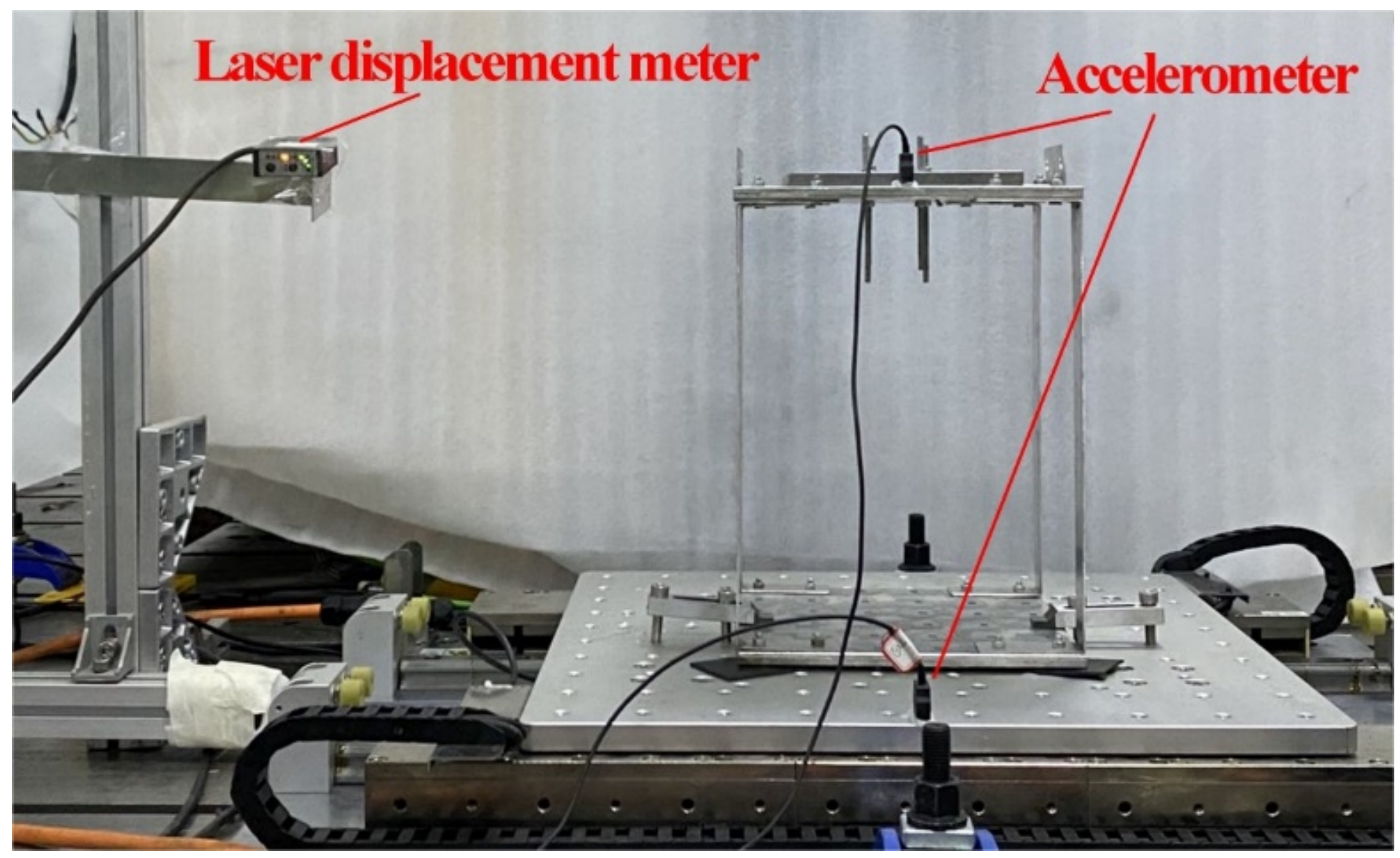

Figure 8

Shaking table RTHS 

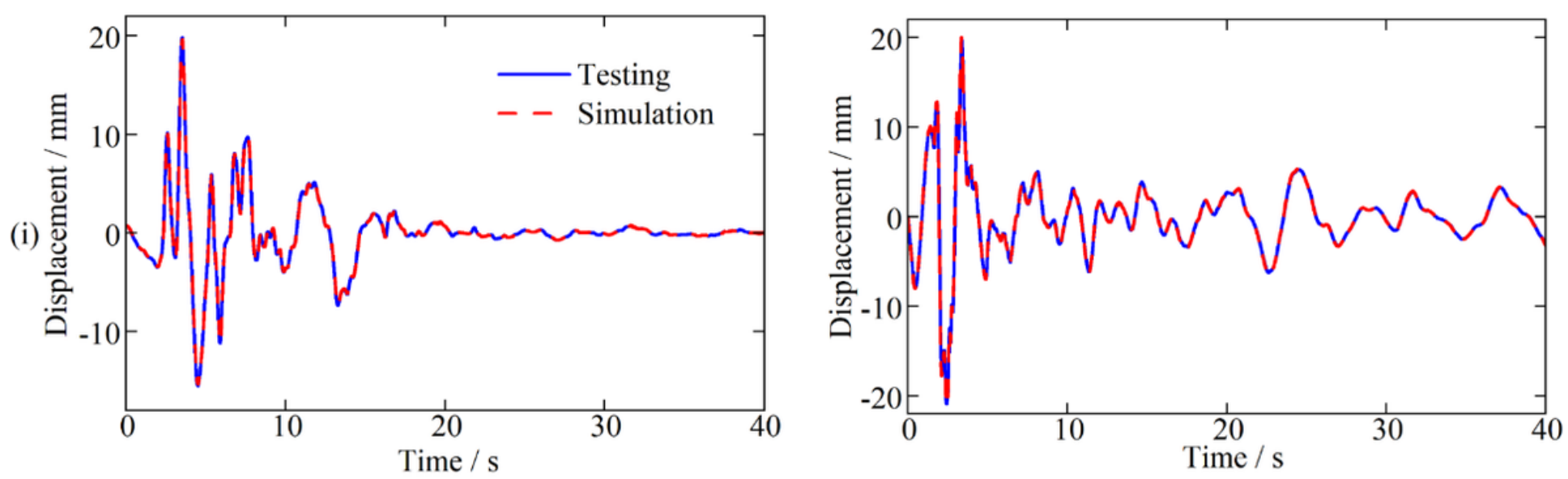

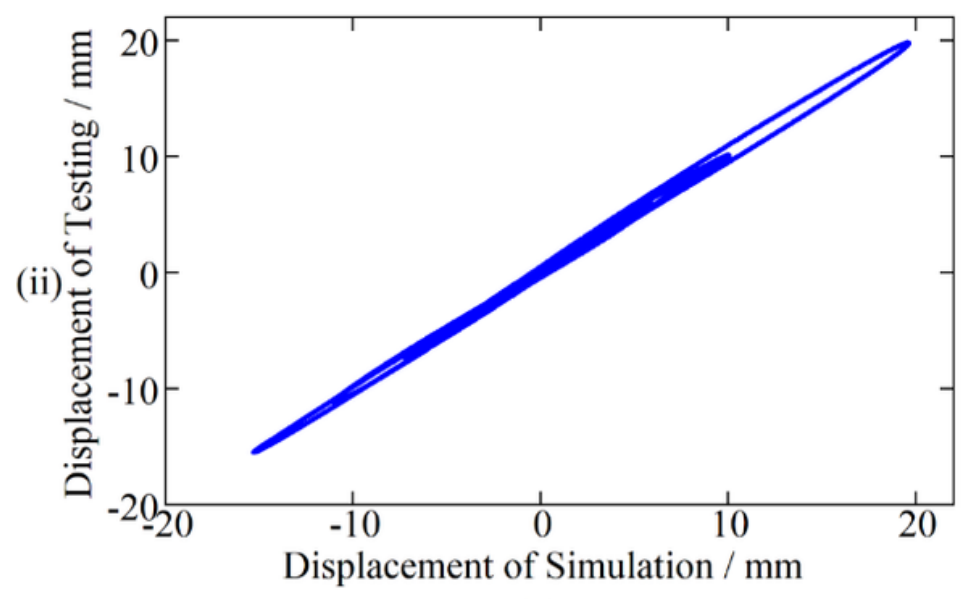

(a)

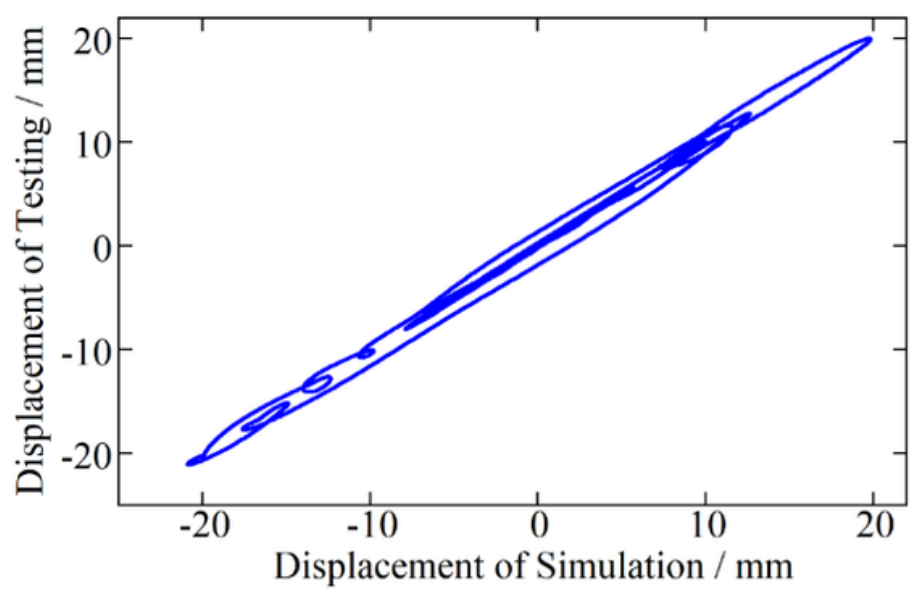

(b)

\section{Figure 9}

The verifications of model parameters under different seismic excitations: (a)Kobe; (b)El-Centro
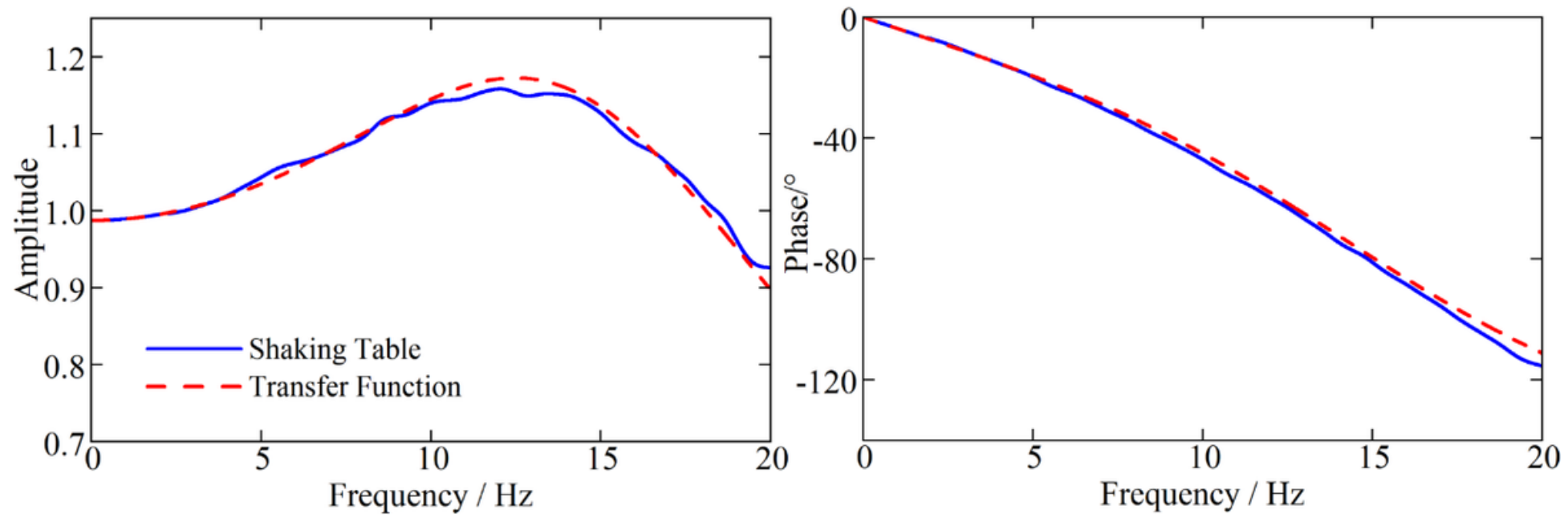

Figure 10

Transfer function of shaking table 


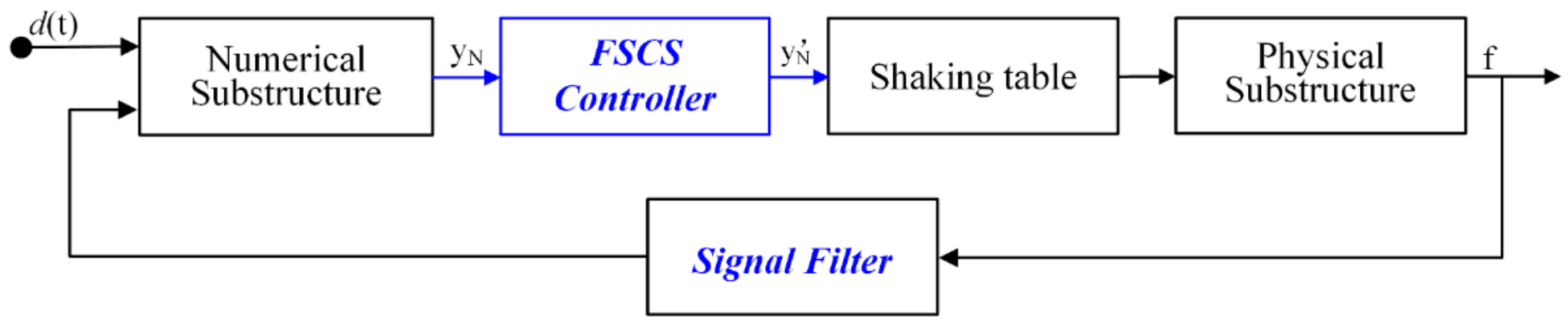

Figure 11

Out-loop control for shaking table
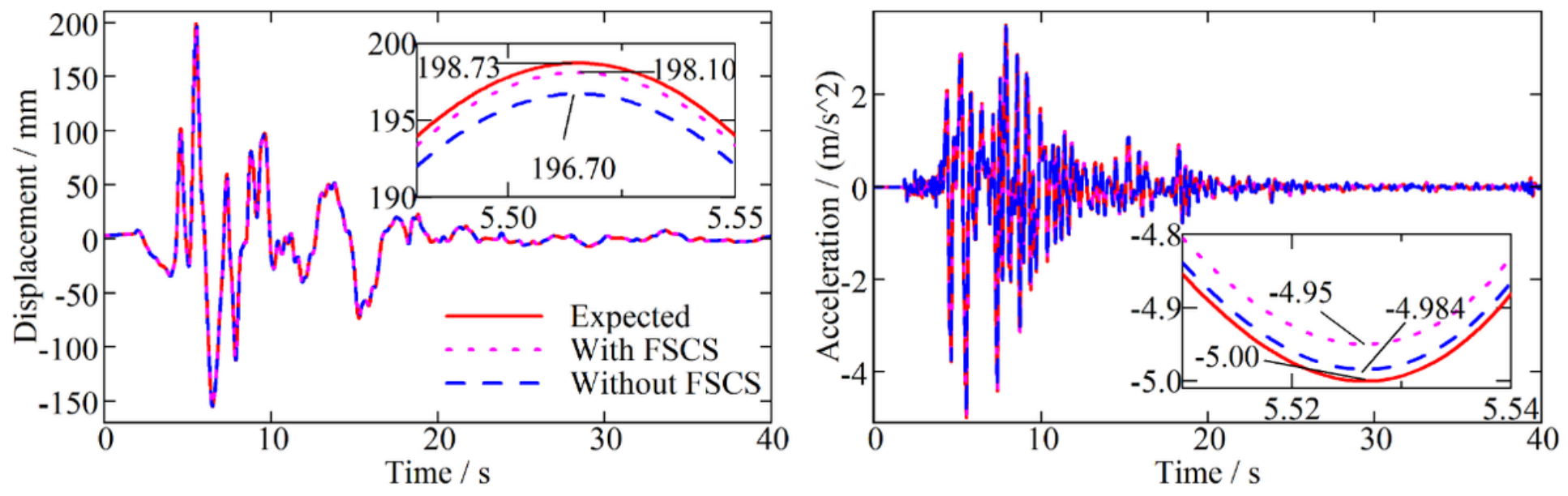

Figure 12

Performance of FSCS controlled shaking table: (a) displacement, (b) acceleration
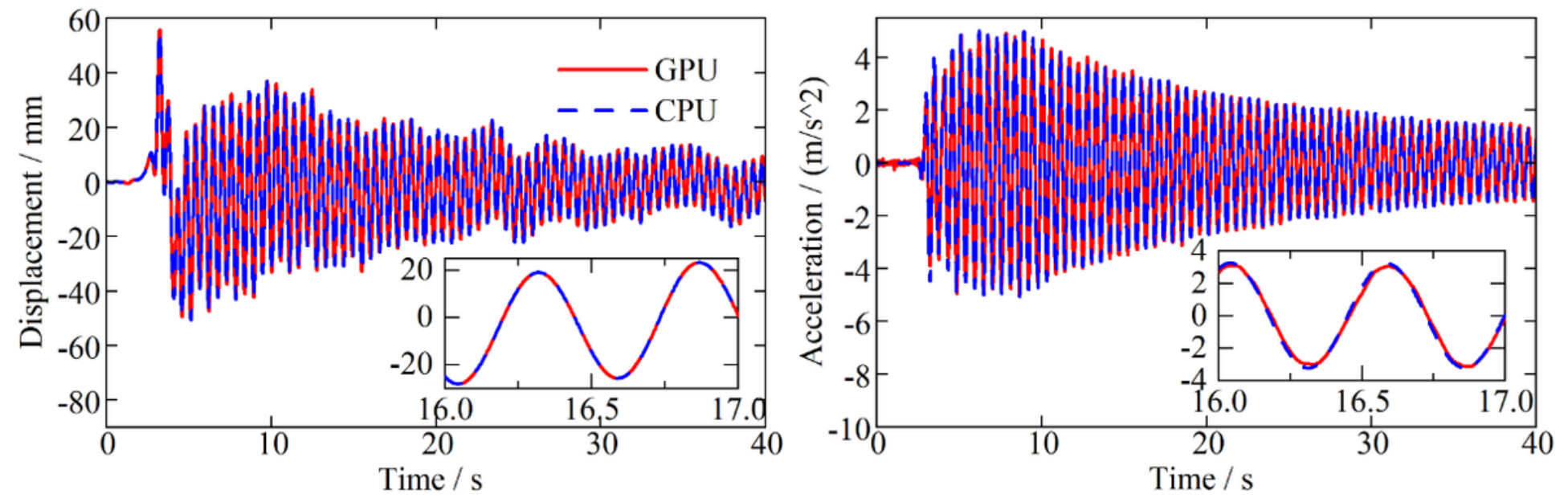

Figure 13

Dynamic response of physical substructure measured from RTHS testing when the numerical model solved by GPU and CPU with 1500 DOFs, double precision and $\Delta \mathrm{t}=4 \mathrm{~ms}$ : (a) displacement, (b) acceleration 


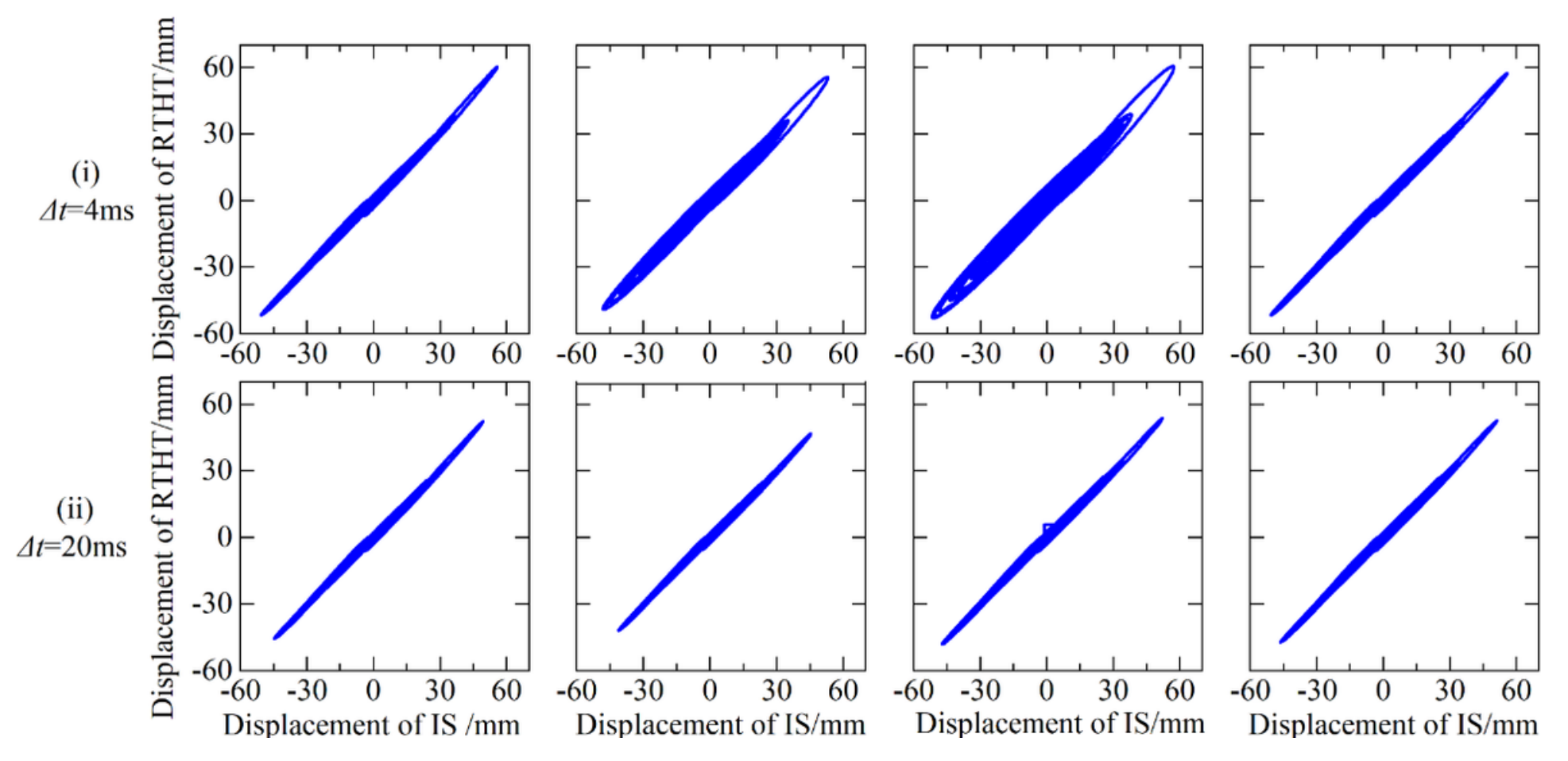

Figure 14

Displacement of physical substructure in RTHS testing and integral simulation (IS)
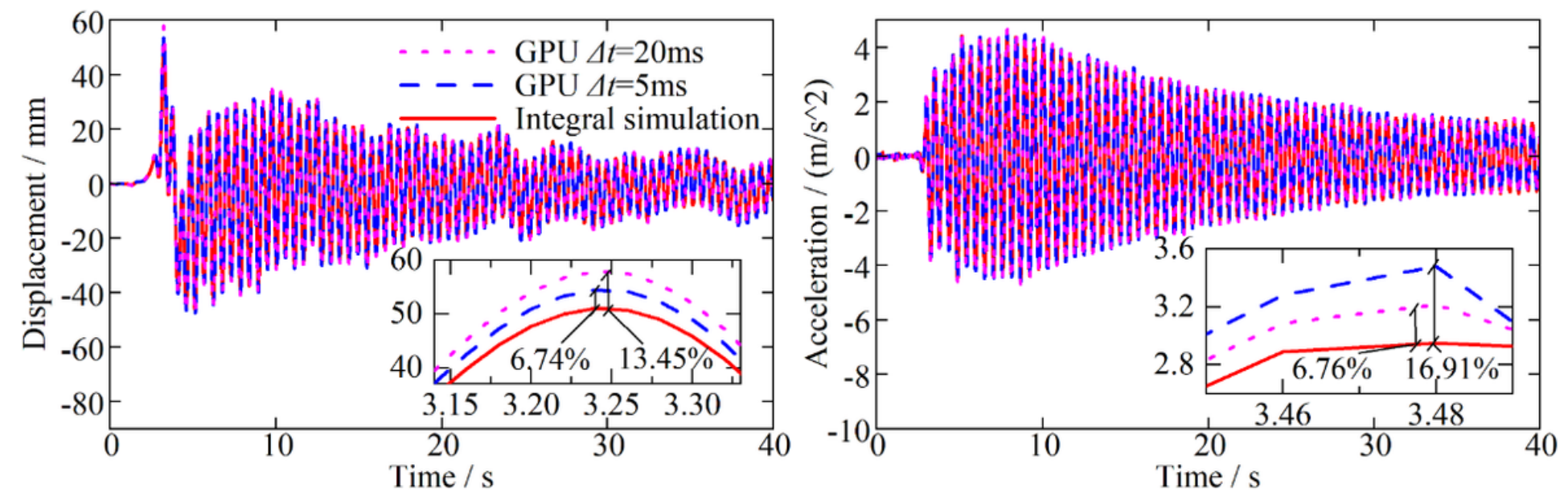

Figure 15

The influence of time step sizes on testing accuracy: (a) displacement, (b) acceleration 